\title{
Productivité et capacité de charge des pâturages naturels au Bénin
}

\author{
D. Agonyissa ${ }^{1}$ B. Sinsin ${ }^{2}$
}

\begin{abstract}
Mots-clés
Pâturage - Biomasse - Capacité de charge - Forêt claire - Savane - Bovin Conduite d'élevage - Bénin.
\end{abstract}

\begin{abstract}
Résumé
Cette étude a été réalisée dans les pâturages soudano-guinéens de la forêt classée de Wari Maro au centre du Bénin. Quatre types de pâturage y ont été distingués sur la base des résultats de relevés phytosociologiques. II s'agit des savanes herbeuses à Schizachyrium sanguineum et Sorghastrum bipennatum des dépressions, des savanes à Andropogon schirensis et $\mathrm{A}$. chinensis, des savanes à Pennisetum unisetum et Chasmopodium caudatum et enfin des jachères à Pennisetum polystachion et Andropogon gayanus. La biomasse produite en fin de saison pluvieuse a varié de 3,45 $\pm 0,50$ à 5,19 $\pm 0,86 \mathrm{t}$ $\mathrm{MS} / \mathrm{ha}$. La biomasse la plus élevée a été obtenue dans le pâturage à Schizachyrium sanguineum et Sorghastrum bipennatum des dépressions. La capacité de charge périodique après le passage des feux de brousse a été estimée à 0,16 unité de bétail tropical par hectare (UBT/ha), soit une charge de saison sèche de 17200 UBT pour une superficie de 107500 ha. En saison humide, la conduite des troupeaux et l'exploitation des pâturages étaient liées à l'évolution de la phytomasse et à la disponibilité en eau dans le système d'élevage extensif en cours dans la zone d'étude.
\end{abstract}

\section{INTRODUCTION}

Les pâturages naturels jouent un rôle important dans l'alimentation du bétail tropical ; ils constituent la base et le plus souvent la totalité des ressources alimentaires des ruminants en élevage extensif (10). Plus de 90 p. 100 de l'énergie consommée par les bovins proviennent des pâturages, mais moins de 10 p. 100 des pâturages sont utilisés d'une façon rationnelle (9).

La République du Bénin dispose de vastes étendues de pâturages naturels estimées entre 7 et 8 millions d'hectares, essentiellement localisées dans les régions septentrionales et centrales du pays (15). La forêt classée de Wari Maro appartient à cet ensemble et, quoique exploitée par les troupeaux transhumants, les données quantitatives sur cet écosystème, indispensables pour une gestion rationnelle des ressources pastorales disponibles et pour l'élaboration d'un plan d'aménagement pour cet écosystème, sont insuffisantes. C'est pour cela que l'étude des pâturages naturels soudanoguinéens de la forêt classée de Wari Maro a été entreprise. Elle s'inscrit dans le programme de recherche du Laboratoire d'Ecolo-

1. Ingénieur agro-pastoraliste chercheur au Laboratoire d'Ecologie appliquée, Faculté des Sciences agronomiques, Université nationale du Bénin, BP 526, Cotonou, Bénin

2. Maître-assistant (même adresse) gie appliquée de la Faculté des Sciences agronomique (Fsa) sur le fonctionnement des écosystèmes pâturés au Bénin. Les objectifs poursuivis étaient les suivants :

- définir la typologie des différents types de pâturage ;

- estimer leur productivité ;

- estimer leur capacité de charge ;

- analyser leur mode d'utilisation.

La forêt classée de Wari Maro appartient au domaine écoclimatique soudano-guinéen et se situe entre $8^{\circ} 45$ et $9^{\circ} 40 \mathrm{~N}$ et entre $2^{\circ}$ et $2^{\circ} 30 \mathrm{E}$. Elle couvre une superficie de 107500 ha et est à cheval sur les sous-préfectures de Bassila et de Tchaourou (figure 1). La forêt se situe donc approximativement au centre du Bénin et constitue pendant la saison sèche un lieu d'accueil des troupeaux transhumants en provenance des pays limitrophes.

La région est couverte par des formations forestières claires, des savanes qui abritent une strate herbacée dominée par de hautes graminées et des galeries forestières. En certains endroits, l'abondance du gymnosperme Encephalartos barteri est un fait unique par sa présence et par son abondance.

La pluviosité moyenne annuelle est de $1200 \mathrm{~mm}$ répartie sur six ou sept mois (avril à octobre). Les pluies précoces de février et (ou) de mars jouent un rôle important dans le démarrage de la végétation, en particulier au niveau de la strate herbacée. Les températures annuelles moyennes sont de 26 ou $27{ }^{\circ} \mathrm{C}$. Les sols sont dans leur majorité sablo-limoneux. 


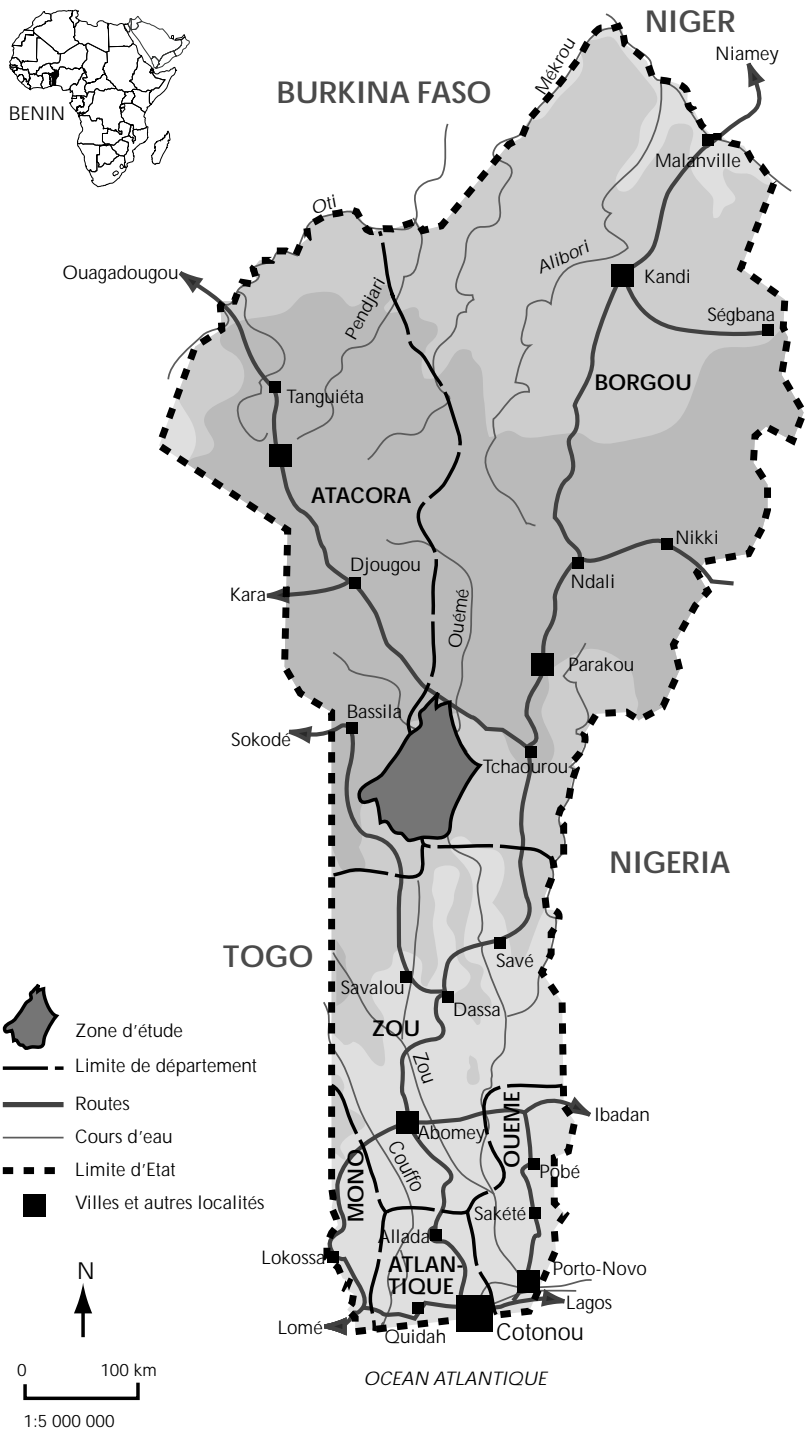

Figure 1 : carte du Bénin montrant la zone d'étude.

\section{METHODOLO GIE}

\section{Typologie}

L'inventaire des pâturages a été réalisé selon la méthode des relevés phytosociologiques (3). Un total de 16 relevés phytosociologiques a été effectué durant la période de maturation des espèces herbacées. L'aire considérée a varié selon l'homogénéité de la végétation. Elle était de $625 \mathrm{~m}^{2}$ environ pour la strate herbacée et de $3600 \mathrm{~m}^{2}$ pour la strate ligneuse. Les listes ainsi établies ont été traitées de manière appropriée (les fréquences relatives (FR) et recouvrements moyens (RM) des espèces ont été utilisés comme premiers critères de classement) en vue d'établir la typologie des pâturages. Ces listes floristiques ont servi au calcul des spectres biologiques bruts et pondérés selon que le recouvrement moyen des spectres a été pris en compte ou non $(8,13)$.
Les types biologiques (TB) utilisés étaient ceux définis par Raunkiaer (2) : les thérophytes (Th), les hémicryptophytes (Hé), les géophytes (Gé), les chaméphytes $(\mathrm{Ch})$ et les phanérophytes $(\mathrm{Ph})$.

Le pourcentage d'espèces communes à deux types de pâturage par rapport aux espèces particulières à chacun des deux a été exprimé par le coefficient de similitude ou coefficient de communauté de Jaccard (14) qui a été utilisé pour comparer les différents types de pâturage. L'expression mathématique de l'indice de Jaccard est :

$\mathrm{I}_{\mathrm{j}}=100 \times \mathrm{c} /(\mathrm{a}+\mathrm{b}-\mathrm{c})$

avec $\mathrm{c}=$ nombre d'espèces communes aux pâturages R1 et R2 ; $\mathrm{a}=$ nombre d'espèces de $\mathrm{R} 1 ; \mathrm{b}=$ nombre d'espèces de $\mathrm{R} 2$.

Selon cette méthode, il a été convenu dans le cadre de cette étude que deux communautés végétales étaient similaires si I était supérieur ou égal à 50 p. 100.

\section{Productivité}

Le matériel végétal a été récolté en fin de période active de végétation par la méthode de coupe rase à l'intérieur de 16 placeaux de $10 \mathrm{~m}$ x $10 \mathrm{~m}$ mis en défens pour toute la durée de l'étude. Ces placeaux étaient répartis sur le terrain sur la base de la géomorphologie en rapport avec les conditions de drainage du sol et selon les principales formations végétales : 3 placeaux dans les jachères sur plateau, 5 placeaux dans les savanes de dépression et 8 placeaux dans les savanes sur plateau. L'intervalle de temps adopté entre deux séries de coupe était de 30 jours. A chaque date de coupe, sept carrés de $1 \mathrm{~m}$ de côté ont été choisis au hasard à l'intérieur de chaque placeau quadrillé et coupés avec un sécateur de jardinier à ras du sol. La phytomasse de la strate herbacée ainsi récoltée a été triée en deux catégories : graminées et autres espèces. Les poids frais du matériel végétal ainsi coupé ont été évalués à l'aide de pesons à ressort de $500 \pm 5 \mathrm{~g}$ et $1000 \pm 10 \mathrm{~g}$ au niveau de chacun des carrés. Pour chacune des deux catégories de plante, graminées et autres espèces, $150 \mathrm{~g}$ ont été prélevés sur le terrain dans des sachets de productivité pour la détermination du poids sec. Un préséchage a été réalisé sur le terrain à l'aide d'un dispositif composé d'un réchaud à gaz, d'une bonbonne de gaz et d'une caisse munie d'un support, conçu à cet effet. Les poids secs de tous les échantillons récoltés, pour l'estimation de la biomasse, ont été notés après séchage à l'étuve à $105{ }^{\circ} \mathrm{C}$ pendant $48 \mathrm{~h}$ jusqu'à poids constant.

\section{Capacité de charge}

Les capacités de charge à différentes périodes de l'année ont été calculées sur la base des phytomasses consommables selon la formule (2) :

$\begin{aligned} & \text { Journées de } \\ & \text { pâture/UBT }\end{aligned}=\frac{\mathrm{k}_{\mathrm{i}} \times \text { quantité de biomasse totale }(\mathrm{kg} \mathrm{MS} / \mathrm{ha})}{6,25 \mathrm{~kg} \mathrm{MS} / \mathrm{UBT} / \mathrm{j}}$

avec $\mathrm{k}_{\mathrm{i}}=\left\{\mathrm{k}_{1}, \mathrm{k}_{2}\right\}$ et $\mathrm{k}_{1}=1 / 3$ pour les savanes $; \mathrm{k} 2=1 / 2$ pour les jachères ; MS $=$ matière sèche ; UBT $=$ unité de bétail tropical.

\section{Utilisation des pâturages}

Des suivis de troupeaux au pâturage ont été réalisés de jour comme de nuit. La pâture nocturne s'est faite généralement entre 3 et $7 \mathrm{~h}$ et la pâture diurne entre 9 et $20 \mathrm{~h}$. A chaque suivi, les formations végétales exploitées ou traversées par les troupeaux ont été notées et les temps de pâture chronométrés. 


\section{Analyse des sols et de la végétation herbacée}

Des échantillons des espèces consommées ont été analysés sur le plan bromatologique. Pour les analyses bromatologiques, la teneur en matière sèche a été déterminée à l'étuve à $80^{\circ} \mathrm{C}$ pendant $24 \mathrm{~h}$. La détermination des cendres a été faite par incinération dans un four à moufle. L'azote a été dosé suivant la méthode de Kjeldahl et la teneur des fourrages en matières azotées totales a été calculée selon la formule (10) :

Matières azotées totales $=\mathrm{N}$ x 6,25

avec $\mathrm{N}=$ teneur en azote

Le dosage de la cellulose brute a été fait suivant la méthode de Weende.

Les méthodes d'analyses chimiques utilisées ont été les méthodes officielles d'analyse de l'AOAC (Association of Official Analytical Chemists).

Des échantillons de sol ont été prélevés entre 0 et $15 \mathrm{~cm}$ de profondeur dans chaque type de pâturage et analysés au Laboratoire des Sciences du sol de la Faculté des Sciences agronomiques de l'Université nationale du Bénin.

La texture et la teneur des sols en matière organique ont été déterminées. Le taux de matière organique a été déterminé par calcination à $700{ }^{\circ} \mathrm{C}$ pendant $2 \mathrm{~h}$. La granulométrie a été effectuée à l'aide de la méthode internationale modifiée par l'utilisation de la pipette de Robinson. La définition de la texture des sols a été faite suivant le diagramme des textures de la FAO.

\section{RESU LTATS ET DISCU SSIO N}

\section{Typologie des pâturages}

\section{Analyse phytosociologique confirmée par les coefficients de Jaccard}

Sur la base des listes floristiques et de l'abondance des espèces dominantes, quatre types de pâturage ont été identifiés :

- le pâturage des savanes herbeuses à Schizachyrium sanguineum et Sorghastrum bipennatum des dépressions ;

- le pâturage à Andropogon schirensis et A. chinensis des savanes arborées sur plateau ;

- le pâturage à Pennisetum unisetum et Chasmopodium caudatum des savanes boisées et des forêts claires ;

- le pâturage à Andropogon gayanus et Pennisetum polystachion des jachères.

Les autres espèces compagnes qui étaient communes dans ces pâturages étaient : Tephrosia elegans, Monocymbium ceresiiforme, Tephrosia nana, Isoberlinia doka, Indigofera paniculata, Vitellaria paradoxa. Le tableau I compare les pâturages deux à deux.

Les valeurs du coefficient de similitude de Jaccard, I, pour les différents types de pâturage comparés deux à deux sont toutes inférieures à 50 p. 100 (tableau I). Les différentes communautés végétales n'étaient donc pas similaires, ce qui a justifié la classification qui a été faite. Ainsi, le coefficient de similitude de Jaccard pour
Tableau I

Degré de communauté entre les différents types de pâturage comparés deux à deux

\begin{tabular}{|c|c|c|c|}
\hline $\begin{array}{l}\text { Pâturages } \\
\text { comparés }\end{array}$ & $\begin{array}{l}\text { Nb. d'espèces } \\
\text { communes }\end{array}$ & $\begin{array}{l}\text { Nb. d'espèces } \\
\text { exclusives }\end{array}$ & $I_{j}(\%)$ \\
\hline $\begin{array}{l}A \\
\text { et } \\
B\end{array}$ & 15 & $\begin{array}{l}11 \\
97\end{array}$ & 12,17 \\
\hline $\begin{array}{l}A \\
\text { et } \\
C\end{array}$ & 9 & $\begin{array}{l}17 \\
87\end{array}$ & 7,96 \\
\hline $\begin{array}{l}A \\
\text { et } \\
D\end{array}$ & 8 & $\begin{array}{l}18 \\
70\end{array}$ & 8,33 \\
\hline $\begin{array}{l}B \\
\text { et } \\
C\end{array}$ & 63 & $\begin{array}{l}49 \\
33\end{array}$ & 43,44 \\
\hline $\begin{array}{l}B \\
\text { et } \\
D\end{array}$ & 62 & $\begin{array}{l}50 \\
16\end{array}$ & 48,43 \\
\hline $\begin{array}{l}C \\
\text { et } \\
D\end{array}$ & 47 & $\begin{array}{l}49 \\
31\end{array}$ & 37,00 \\
\hline
\end{tabular}

$\mathrm{A}=$ pâturage à Schizachyrium sanguineum et Sorghastrum bipennatum

$\mathrm{B}=$ pâturage à Andropogon schirensis et $A$. chinensis

$\mathrm{C}=$ pâturage à Pennisetum unisetum et Chasmopodium caudatum

$\mathrm{D}=$ pâturage à Pennisetum polystachion et Andropogon gayanus

les pâturages à Schizachyrium sanguineum et Sorghastrum bipennatum, et à Andropogon schirensis et A. chinensis a donné une valeur de 12,2 p. 100. Les auteurs ont déduit de ce résultat que le pâturage à Schizachyrium sanguineum et Sorghastrum bipennatum, et le pâturage à Andropogon schirensis et A. chinensis étaient différents sur le plan de leur composition floristique.

\section{Types biologiques}

Les spectres biologiques des pâturages sont présentés aux figures $2,3,4$ et 5 .

La figure 2 montre les spectres biologiques du pâturage à Schizachyrium sanguineum et Sorghastrum bipennatum des dépressions. Les espèces hémicryptophytes ont dominé alors que les thérophytes étaient les plus abondantes. Les deux espèces les plus dominantes étaient Schizachyrium sanguineum et Sorghastrum bipennatum. Elles ont contribué en moyenne au spectre pondéré pour 70 p. 100 et ont défini la physionomie du pâturage. Le tapis herbacé était exempt de tout ligneux.

Sur la figure 3 (pâturage de savanes arborées sur plateau), on note la prépondérance des phanérophytes par rapport aux thérophytes. En tenant compte du recouvrement moyen, c'étaient néanmoins les thérophytes qui ont alors dominé par rapport aux phanérophytes et aux hémicryptophytes.

Sur la figure 4 (pâturage de savanes boisées et de forêts claires), on peut constater l'abondance et la dominance des phanérophytes qui avaient le spectre brut le plus élevé, dépassant celui des théro- 
phytes. L'abondance de ces phanérophytes ajoutée au recouvrement élevé de la strate arborescente (45 p. 100) expliquent le faible éclairement que l'on note au niveau de la strate herbacée. La figure 5 montre la répartition des spectres biologiques du pâturage à Andropogon gayanus et Pennisetum polystachion des groupements post-culturaux. Les phanérophytes présents sous forme de recrus étaient les plus abondants. En revanche, c'étaient les thérophytes qui étaient les plus dominants dépassant les phanérophytes.
Il est à noter que les phanérophytes qui ont été recensés dans la strate herbacée au cours des relevés étaient constitués de jeunes plants issus de semences d'arbres qui ont soit survécu au feu de brousse de la saison sèche récente, soit échappé au broutage. Les fréquences assez élevées des phanérophytes au niveau de la strate herbacée ainsi que leur fort pourcentage de recouvrement étaient très révélateurs de l'état de dégradation des pâturages, caractérisé par la régression des graminées hémicryptophytes (13).

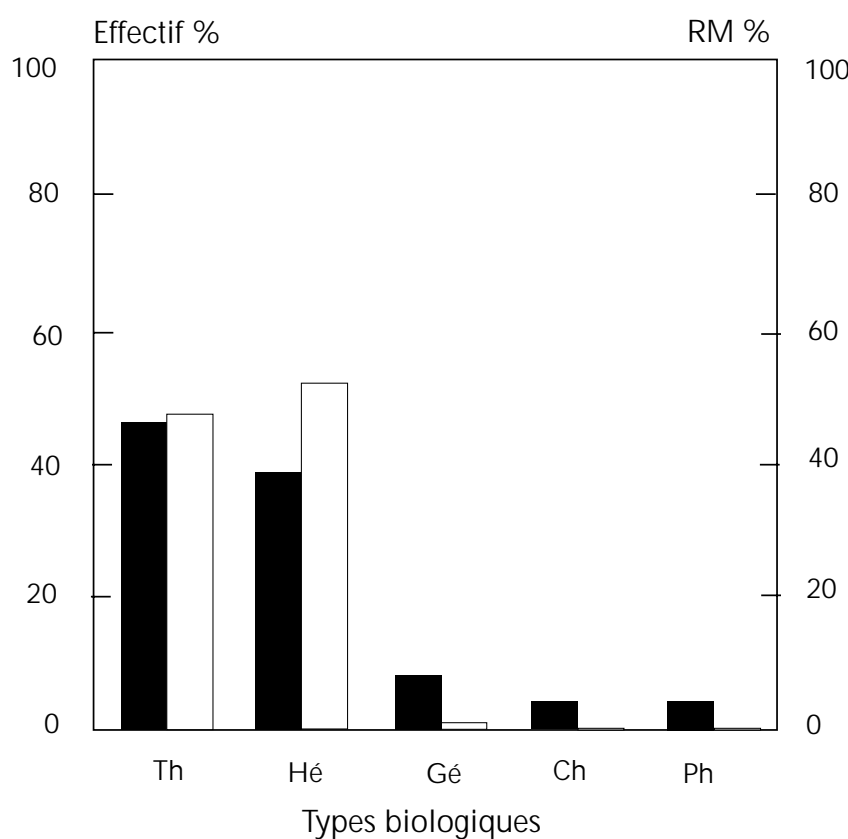

Spectre brut

Figure 2 : spectres biologiques du pâturage à Schizachyrium sanguineum et Sorghastrum bipennatum.

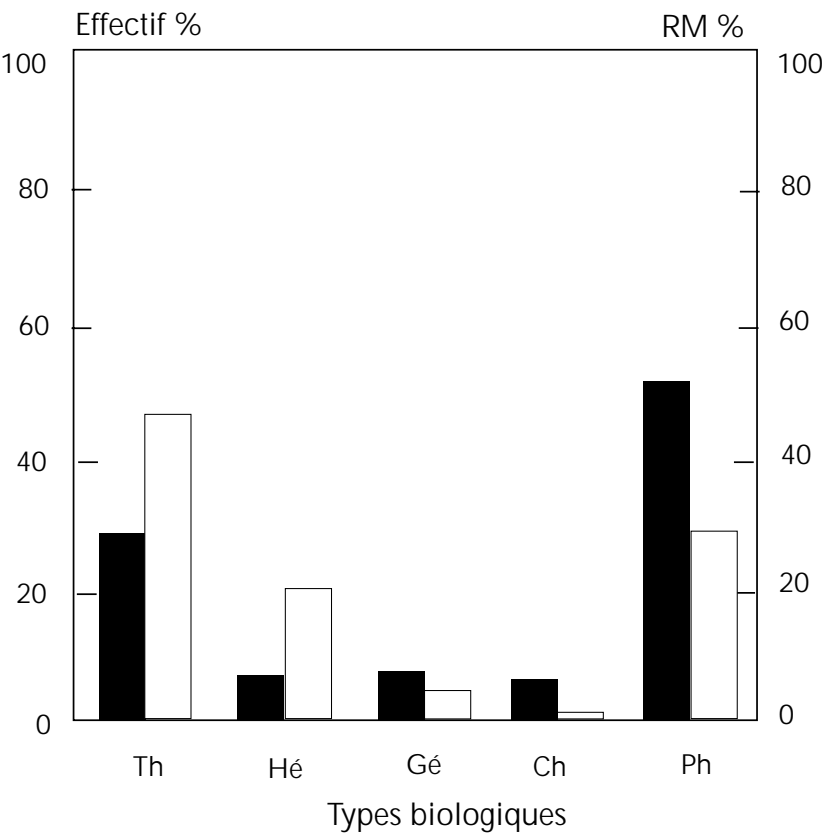

Spectre brut Spectre pondéré

Figure 3 : spectres biologiques du pâturage à Andropogon schirensis et $\mathrm{A}$. chinensis.

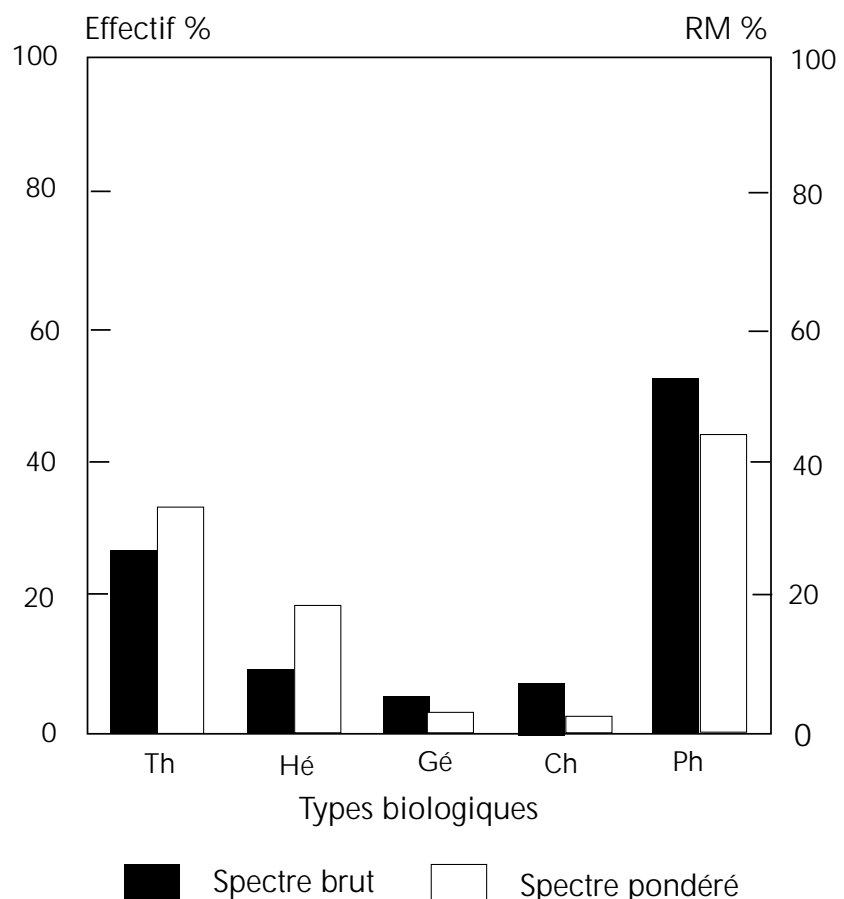

Figure 4 : spectres biologiques du pâturage à Pennisetum unisetum et Chasmopodium caudatum.

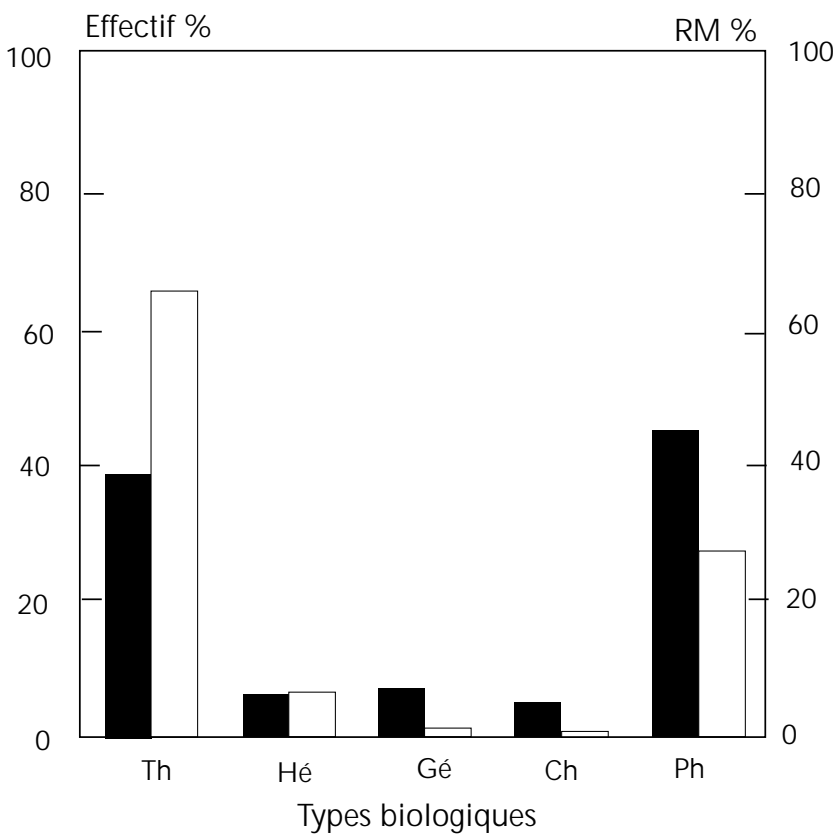

Spectre brut

Spectre pondéré

Figure 5 : spectres biologiques du pâturage à Pennisetum polystachion et Andropogon gayanus. 


\section{Phytomasse}

La production de matière sèche était très variable d'un type de pâturage à l'autre. La phytomasse a varié entre 3,4 et 5,2 t MS/ha. La disponibilité en eau du sol, directement liée à la position topographique, a joué un rôle important $(5,8,12)$. Ainsi, la savane herbeuse à Schizachyrium sanguineum et Sorghastrum bipennatum située dans les bas-fonds et donc relativement humide, avait la phytomasse la plus élevée, soit 5,2 t MS/ha. De plus, le sol avait une forte teneur en éléments fins (tableau II).

Le tableau III présente les résultats, exprimés en poids de matière sèche au pic de biomasse.

D'une manière générale, la phytomasse herbacée est d'autant plus élevée que le sol est riche en fractions fines et que le couvert ligneux est faible. La valeur de la phytomasse la plus faible,
3,4 t MS/ha, était celle du pâturage à Pennisetum unisetum et à Chasmopodium caudatum. Ce pâturage pousse sur plateau, sous un couvert ligneux important (45 p. 100). Ceci limite vraisemblablement la densité des touffes quoique Pennisetum unisetum ait une tendance sciaphile en zone soudanienne (13). Cette différence de la phytomasse selon la situation topographique est attribuable à la composition floristique en rapport avec les conditions du sol (approvisionnement en eau, caractères physiques et chimiques des sols) et à la structure des groupements végétaux herbacés (11).

D’une façon générale, les données de phytomasse de la zone étudiée s'inscrivent dans le même ordre de grandeur que celles obtenues par différents auteurs à des latitudes similaires dans les régions soudano-guinéennes : 3 à $10 \mathrm{t} \mathrm{MS} / \mathrm{ha}$ à Ouango-Fitini à $9^{\circ} 35 \mathrm{~N}(5)$ et 3,3 à $7,4 \mathrm{t} \mathrm{MS} / \mathrm{ha}$ au Nigeria (1).

\section{Tableau II}

Constitution texturale, teneur en matière organique et texture des sols des différents types de pâturage

\begin{tabular}{|c|c|c|c|c|c|c|}
\hline \multirow[t]{2}{*}{ Pâturage à } & \multirow[t]{2}{*}{$\begin{array}{l}\text { Humidité } \\
\text { (\%) }\end{array}$} & \multirow[t]{2}{*}{$\begin{array}{l}\text { MO } \\
(\%)\end{array}$} & \multicolumn{4}{|c|}{$\begin{array}{c}\text { Granulométrie } \\
(\%)\end{array}$} \\
\hline & & & Argile & $\begin{array}{l}\text { Limon } \\
\text { fin }\end{array}$ & $\begin{array}{l}\text { Limon } \\
\text { grossier }\end{array}$ & Sable \\
\hline $\begin{array}{l}\text { Schizachyrium sanguineum } \\
\text { et Sorghastrum bipennatum }\end{array}$ & 1,05 & 3,23 & 12,66 & 14,59 & 6,48 & 61,53 \\
\hline $\begin{array}{l}\text { Andropogon schirensis } \\
\text { et } A \text {. chinensis }\end{array}$ & 0,79 & 2,70 & 7,10 & 9,25 & 5,04 & 74,52 \\
\hline $\begin{array}{l}\text { Pennisetum unisetum } \\
\text { et Chasmopodium caudatum }\end{array}$ & 0,89 & 2,68 & 6,18 & 7,09 & 4,85 & 77,48 \\
\hline $\begin{array}{l}\text { Pennisetum polystachion } \\
\text { et Andropogon gayanus }\end{array}$ & 0,57 & 2,13 & 5,62 & 5,78 & 2,16 & 83,36 \\
\hline
\end{tabular}

$\mathrm{MO}=$ matière organique

Tableau III

Comparaison des phytomasses des différents types de pâturage selon le test de Newman-Keuls au seuil de 5 p. 100

\begin{tabular}{|c|c|c|c|c|}
\hline Types de pâturage & $\begin{array}{l}\text { Moyennes } \\
\left(\mathrm{g} \mathrm{MS} / \mathrm{m}^{2}\right)\end{array}$ & & $\begin{array}{c}\text { Groupes } \\
\text { homogènes }\end{array}$ & \\
\hline \multicolumn{5}{|l|}{ Variable : graminées } \\
\hline $\begin{array}{l}\text { Pâturage à Schizachyrium sanguineum } \\
\text { et Sorghastrum bipennatum }\end{array}$ & 490,43 & A & & \\
\hline $\begin{array}{l}\text { Pâturage à Andropogon schirensis } \\
\text { et } A \text {. chinensis }\end{array}$ & 480,99 & A & & \\
\hline $\begin{array}{l}\text { Pâturage à Pennisetum unisetum } \\
\text { et Chasmopodium caudatum }\end{array}$ & 405,65 & & B & \\
\hline $\begin{array}{l}\text { Pâturage à Pennisetum polystachion } \\
\text { et Andropogon gayanus }\end{array}$ & 340,69 & & & C \\
\hline \multicolumn{5}{|l|}{ Variable : biomasse totale } \\
\hline $\begin{array}{l}\text { Pâturage à Schizachyrium sanguineum } \\
\text { et Sorghastrum bipennatum }\end{array}$ & 519,95 & A & & \\
\hline $\begin{array}{l}\text { Pâturage à Andropogon schirensis } \\
\text { et } A \text {. chinensis }\end{array}$ & 511,96 & A & & \\
\hline $\begin{array}{l}\text { Pâturage à Pennisetum unisetum } \\
\text { et Chasmopodium caudatum }\end{array}$ & 411,37 & & $B$ & \\
\hline $\begin{array}{l}\text { Pâturage à Pennisetum polystachion } \\
\text { et Andropogon gayanus }\end{array}$ & 345,32 & & & C \\
\hline
\end{tabular}


Les résultats de l'analyse statistique des données de productivité sont présentés dans le tableau III en distinguant la biomasse des graminées de la biomasse totale au niveau des quatre types de pâturage. Les résultats de l'analyse de variance révèlent une différence significative au seuil de 5 p. 100 entre les types de pâturage.

L'analyse de ces résultats permet de distinguer trois types de pâturage du point de vue de la productivité de phytomasse. Ceci est à la fois valable pour les graminées et pour la biomasse totale. Ainsi, les phytomasses des pâturages à Schizachyrium sanguineum et Sorghastrum bipennatum des savanes herbeuses de dépression, et à Andropogon schirensis et $A$. chinensis des savanes arborées sur plateau ne présentent pas de différence significative au seuil de 5 p. 100.

La faible productivité des pâturages de savanes à Pennisetum unisetum et Chasmopodium caudatum et des jachères à Pennisetum polystachion et Andropogon gayanus était liée à la pauvreté chimique de leurs sols, notamment en matière organique et en argile.

\section{Capacité de charge}

La capacité de charge en biomasse animale est proportionnelle à la biomasse végétale. Les capacités de charge théorique des deux principales formations végétales (jachère et savane) étaient respectivement de $0,86 \mathrm{UBT} / \mathrm{ha} / \mathrm{an}$ soit $1,16 \mathrm{ha} / \mathrm{UBT} / \mathrm{an}$ et de 0,60 $\mathrm{UBT} / \mathrm{ha} / \mathrm{an}$ soit 1,66 ha/UBT/an au pic de biomasse.

Pendant la saison pluvieuse, la valeur des pâturages était en général satisfaisante et la phytomasse produite pouvait supporter des charges de l'ordre de 1,93 UBT/ha. A cette période, le taux de charge de la région devient faible par rapport à la disponibilité de fourrages, ce qui se traduit sur le terrain par une sous-exploitation des pâturages (11).

$\mathrm{Au}$ début de la saison sèche, les touffes de graminées qui n'étaient pas broutées se sont lignifiées et 90 p. 100 de la biomasse produite a été détruite par les feux de brousse.

Pendant la saison sèche (novembre à mars) il a fallu $6,25 \mathrm{ha} / \mathrm{UBT}$, soit une charge de 17200 UBT pour toute la forêt classée, si l'on considère qu'il ne reste qu'un dixième de la biomasse après le passage des feux de brousse.

Ces résultats expliquent les difficultés que rencontraient les éleveurs dans leur recherche de fourrage pour leur troupeau pendant la saison sèche, la zone d'étude ayant constitué pendant cette période un lieu d'accueil des troupeaux transhumants en provenance des pays limitrophes du Bénin. Les troupeaux nigérians allaient pour la plupart au Togo pour rejoindre l'important marché à bétail d'Anié situé à une cinquantaine de kilomètres de Tchèti (sous-préfecture de Savalou) à la même latitude que la zone d'étude.
Malgré l'occupation de la forêt classée de Wari Maro par les troupeaux domestiques, la faune sauvage y est relativement bien représentée (7). La flore est à la fois aussi bien fournie au niveau de la strate arborescente qu'herbacée. Ceci est un atout pour la production animale au sens large. En effet, les plantes non consommées par les bovins domestiques peuvent être exploitées par certains ruminants (6). Il peut en résulter un potentiel de charge plus élevé avec la présence conjointe d'ongulés domestiques et sauvages.

\section{Composition chimique de la strate herbacée}

Le tableau IV présente les résultats d'analyse bromatologique de quelques espèces constituantes des pâturages étudiés. D'une manière générale, les espèces analysées ont présenté une faible valeur fourragère, fonction de l'anatomie des feuilles, du poids des chaumes et de la démographie foliaire de chaque espèce $(2,5,13)$. L'analyse des résultats a révélé que la teneur en matières azotées totales (MAT) des espèces fourragères était proportionnelle à l'indice de qualité $(4,13)$. L'espèce Andropogon gayanus qui avait la plus forte teneur en MAT (2,85 p. 100) parmi les espèces analysées était manifestement celle qui était la plus recherchée par les bovins. De même l'espèce Sorghastrum bipennatum, avec une faible teneur en MAT (0,13 p. 100), était peu broutée.

Cette concordance entre les indices de qualité (définis de manière empirique) et les teneurs en nutriments montre bien que le choix d'une espèce fourragère par les animaux est souvent en corrélation positive avec sa valeur alimentaire.

\section{Exploitation traditionnelle des pâturages}

Les suivis périodiques des troupeaux bovins à travers les différentes formations végétales ont permis une meilleure connaissance du mode d'exploitation des pâturages de la région d'étude pendant la saison pluvieuse. La figure 6 montre comment les différentes formations végétales ont été exploitées.

D’une façon générale, les jachères étaient les milieux les plus exploités pendant la saison des pluies. Le temps de pâture était plus élevé dans les jachères que dans les savanes de juin à septembre avec un écart maximum de 21 p. 100 en août (période de floraison d'Hyparrhenia involucrata qui dominait la majorité des pâturages). Avant la floraison, cette espèce végétale était bien appétée et l'essentiel de l'alimentation des animaux se faisait au niveau de la strate herbacée. En juin, les distances parcourues par les éleveurs pour nourrir le bétail ne dépassaient guère $5 \mathrm{~km} / \mathrm{jour}$. Les jachères constituaient les milieux les plus fréquentés de par leur situation assez proche des villages. Du point de vue structural,

\section{Tableau IV}

Résultats d'analyse de quelques espèces dominantes des différents types de pâturage dans la zone d'étude (dates de récolte : 28-30/08/95)

$\begin{array}{lcccc}\text { Espèces } & \begin{array}{c}\text { MS } \\ (\%)\end{array} & \begin{array}{c}\text { Cendre } \\ (\%)\end{array} & \begin{array}{c}\text { Matières } \\ \text { azotées } \\ \text { totales } \\ (\%)\end{array} & \begin{array}{c}\text { Cellulose } \\ \text { brute } \\ (\%)\end{array} \\ \text { Hyparrhenia involucrata } & & 7,9 & 0,9 & 44,33 \\ \text { Schizachyrium sanguineum } & 90,7 & 8,8 & 1,9 & 41,16 \\ \text { Andropogon gayanus } & 89,3 & 6,4 & 2,8 & 41,33 \\ \text { Andropogon schirensis } & 89,4 & 10,7 & 0,9 & 43,09 \\ \text { Sorghastrum bipennatum } & 90,5 & 10,2 & 0,1 & 39,83\end{array}$


Temps de pâture journalière \%

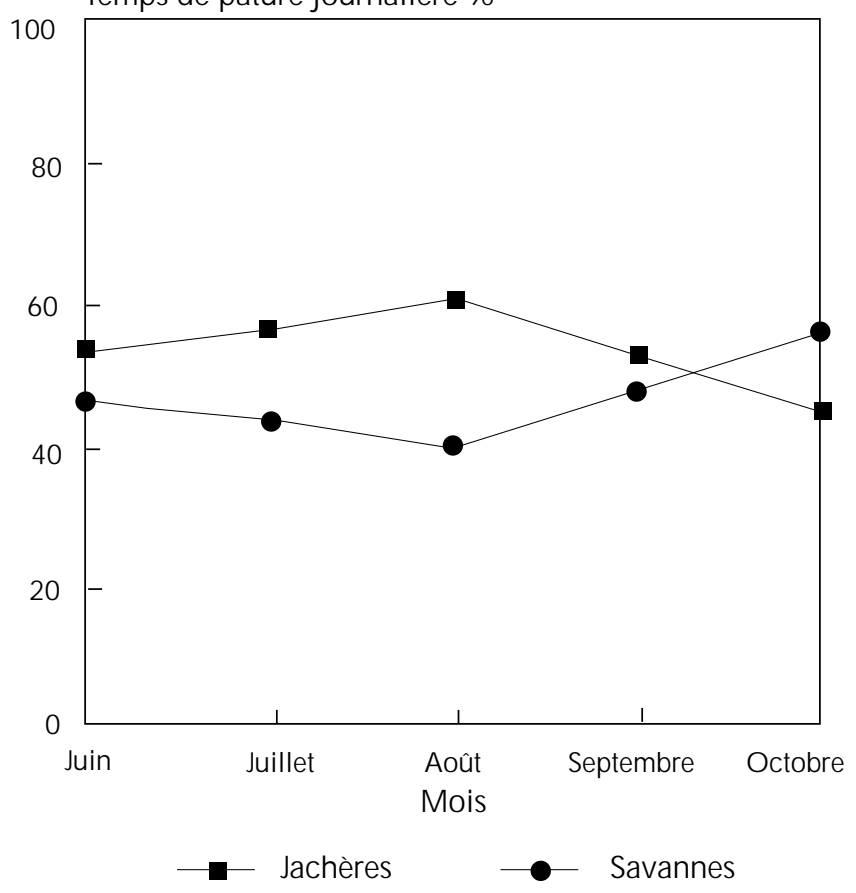

Figure 6 : répartition des temps de pâture journalière dans les savanes et les jachères.
La pâture nocturne est un fait courant et elle s'explique pour diverses raisons. Elle a lieu habituellement entre 3 et $7 \mathrm{~h}$ du matin. Elle présente, selon les agro-éleveurs, un double avantage : d'une part, l'abreuvement des animaux n'est pas indispensable grâce à la présence de la rosée sur les espèces fourragères prélevées et à la douceur de la température de la nuit ; d'autre part, les animaux pâturent aisément du fait de l'absence de mouches tsé-tsé pendant la nuit. En définitive, la pâture nocturne pratiquée par les agro-éleveurs est liée aux contraintes climatiques et écologiques du milieu.

En octobre, les graminées qui ont constitué les espèces les plus exploitées étaient dans leur majorité lignifiées. Mais, paradoxalement, les savanes de la forêt classée de Wari Maro étaient les milieux les plus exploités par les troupeaux autochtones et transhumants qui y ont trouvé des pâturages entretenus par la fréquentation régulière. Les animaux se sont toujours alimentés au niveau de la strate herbacée au cours de ce mois. Le Lingué Afzelia africana et le Caïlcédrat Khaya senegalensis qui sont les ligneux les plus consommés pendant la saison sèche (11) n'étaient pas jusque là émondés.

L'arrivée de la saison sèche, avec l'allumage généralisé des feux de brousse, a limité la disponibilité fourragère de la strate herbacée. Les résidus de récolte auxquels s'est ajouté le fourrage des ligneux fourragers, malgré leur richesse en protéine et en énergie fourragère, ne sont pas arrivés à résoudre le problème d'alimentation pour les troupeaux autochtones et pour les transhumants étrangers à la région : la densité des arbres fourragers était trop faible face à l'afflux grandissant des troupeaux et à leurs besoins d'entretien. le pâturage des jachères est demeuré assez ras durant une bonne partie de la période humide. Dans les dépressions, les graminées étaient au début de la montaison et leur taille ne dépassait guère $50 \mathrm{~cm}$, ce que préféraient généralement les bovins. Pendant cette première phase de la période humide (juin-juillet), les espèces les plus consommées dans les jachères étaient des graminées telles que Andropogon gayanus, Brachiaria falcifera, Hyparrhenia involucrata, etc. Dans les savanes de dépression proches des lieux d'abreuvement des animaux, les espèces comme Schizachyrium sanguineum et Sorghastrum bipennatum étaient appétées. A partir de la fin du mois de juillet, les bovins ont aussi pâturé la nuit. Ce phénomène de pâture nocturne est particulièrement remarquable chez les bovins de race Zébu.

$\mathrm{Au}$ mois d'août, diverses dicotylédones fourragères ont été consommées, notamment les légumineuses fourragères telles que Tephrosia bracteolata, Indigofera paniculata, Chamaecrista mimosoides, Crotalaria microcarpa, etc. Les autres espèces appétées étaient Tridax procumbens, Hibiscus asper, etc.

En septembre, les préférences alimentaires n'étaient pas très différentes de celles du mois de juillet. Le problème d'abreuvement ne s'est guère posé. Dans les savanes où les graminées ont dominé la strate herbacée, les espèces ont été consommées à des stades de développement et à des degrés divers. Les espèces les plus consommées étaient les graminées telles que Andropogon gayanus, Andropogon schirensis, A. chinensis et Brachiaria falcifera. Les jachères ont été exploitées en raison de la qualité de leur fourrage due essentiellement aux espèces telles que Pennisetum polystachion, Andropogon gayanus, Chasmopodium caudatum et Tridax procumbens. Les graminées ont été préférées pendant cette période dans les jachères comme dans les savanes et ont constitué la majeure partie de la phytomasse ingérée.

\section{$\square$ CONCLUSION}

L'analyse des résultats phytosociologiques des pâturages naturels a permis l'établissement d'une typologie des pâturages soudanoguinéens de la forêt classée de Wari Maro. Quatre types de pâturage se sont différenciés :

- le pâturage à Schizachyrium sanguineum et Sorghastrum bipennatum des dépressions à sols riches en éléments fins et ayant la phytomasse la plus élevée ;

- le pâturage à Andropogon schirensis et A. chinensis des savanes arborées ;

- le pâturage à Pennisetum unisetum et Chasmopodium caudatum des savanes boisées et des forêts claires ayant la phytomasse la plus faible sous un couvert ligneux ;

- le pâturage à Pennisetum polystachion et Andropogon gayanus des jachères.

Pendant la saison pluvieuse, le pâturage à Pennisetum polystachion et Andropogon gayanus des jachères était le plus exploité. Après le passage des feux de brousse de saison sèche, la capacité de charge saisonnière de la zone est devenu très faible et il fallait plus de six hectares pour couvrir les besoins d'entretien d'une UBT. Cette faible capacité de charge saisonnière explique les difficultés que rencontraient les éleveurs dans leur recherche de fourrage pour leurs troupeaux pendant la saison sèche. Le déplacement des troupeaux vers les savanes, ou transhumance, est devenu une nécessité en saison sèche et c'étaient les aires protégées qui, dans la majorité des cas, ont subi de fortes pressions de pâture par les troupeaux domestiques. 


\section{BIBLIO GRAPHIE}

1. AFO LAYAN T.A., 1978. Grass biomass production in Northern Guinea savanna ecosytem. O ecol. Plant., 13: 375-386.

2. BOUDET G., 1984. Manuel sur les pâturages tropicaux et les cultures fourragères, $4^{e}$ ed. Paris, France, Ministère des relations extérieures, coopération et développement, 266 p. (Coll. Manuels et précis d'élevage)

3. BRAUN-BLANQUET J., 1932. Plant sociology. The study of plant communities. N ew York, USA, London, UK, Mc Gray Hill, 439 p.

4. DAGET P., POISSONET J., 1971. Une méthode d'analyse phytologique des prairies. Critères d'application. Ann. Agron., 22 : 5-41.

5. FOURNIER A., HOFFMANN D., DEVINEAU J.L., 1982. Variations de la phytomasse herbacée le long d'une toposéquence en zone soudanoguinéenne, O uango-Fitini (Côte d'Ivoire). Bull. IFAN, 44 (1-2) : 71-77.

6. FRITZ H., DUNCAN P., 1993. Large herbivores in rangelands. Nature, 364: 292-293.

7. HEYMANS J.C., PETIT J.M., 1985. Etude et aménagement de la forêt classée des Monts Kouffé (République du Bénin). Note préliminaire. Tropicultura, $3: 88-92$

8. LE HOUEROU H.N., 1989. The grazing land ecosystems of the African Sahel. Berlin, Germany, Springer-Verlag, $228 \mathrm{p}$.

9. PAGOT J., 1985. L'élevage en pays tropicaux. Paris, France, Maisonneuve et Larose, ACCT, $526 \mathrm{p}$.
10. RIVIERE R., 1977. Manuel d'alimentation des ruminants domestiques en milieu tropical. Paris, France, Ministère de la coopération, $523 \mathrm{p}$. (Coll. Manuels et précis d'élevage $n^{\circ} 9$ )

11. SIN SIN B., 1991. Exploitation des pâturages naturels dans un système traditionnel d'élevage bovin dans le périmètre Nikki-Kalalé. In : Actes IVe Congr. int. des terres de parcours, Montpellier, France, 2226 avril 1991, p. 660-662.

12. SINSIN B., 1991. Influence de la topographie sur la production pluviale des pâturages herbacés des savanes soudaniennes du NordBénin. In : Actes Sémin. int. Gestion agroclimatique des précipitations, Bamako, Mali, 9-13 décembre 1991

13. SINSIN B., 1993. Phytosociologie, écologie, valeur pastorale, production et capacité de charge des pâturages naturels du périmètre Nikki-Kalalé au Nord-Bénin. Thèse Doct., U niversité Libre de Bruxelles, Belgique, $390 \mathrm{p}$.

14. SINSIN B., OUMOROU M., OGOUBYI V., 1996. Les faciès à Andropogon pseudapricus des groupements post-culturaux et des savanes arbustives du Nord-Bénin : dissemblance floristique et caractères communs. In : Van der Maesen L.J.G., Van der Burgt, Van Medenbach de Rooy J.M., eds., The biodiversity of African plants. D ordrecht, The Netherlands, Kluwer Academic Publishers, p. 231-238.

15. TCHIW AN OU M., 1994. Les Fulbé éleveurs face aux nouvelles stratégies de gestion participative des ressources forestières au Bénin. $\mathrm{Na}$ ture Info Bénin (2-3) : 14-15.

Reçu le 12.8.97, accepté le 15.12 .98

\section{Summary}

Agonyissa D., Sinsin B. Productivity and carrying capacity of natural grassland in Benin

This study was carried out in the Sudano-Guinean grassland of $W$ ari $M$ aro protected forest in the central region of Benin. Four pasture types were distinguished based on the results of phytosociological relevés. They were Schizachyrium sanguineum and Sorghastrum bipennatum of grass savanna sunken sites, Andropogon schirensis and A. chinensis savannas, Pennisetum unisetum and Chasmopodium caudatum savannas and, finally, Pennisetum polystachion and Andropogon gayanus fallows. At the end of the rainy season, biomass varied from $3.45 \pm 0.50$ to $5.19 \pm 0.86 \mathrm{t} \mathrm{DM} / \mathrm{ha}$. Schizachyrium sanguineum and Sorghastrum bipennatum in sunken pastures produced the highest biomass. The periodic carrying capacity of pastures after bush fire was estimated as 0.16 tropical livestock unit per hectare (TLU/ha), or a dry season capacity of 17,200 TLU for 107,500 ha. In the wet season cattle management and grassland exploitation depended on phytomass and water availability in the extensive husbandry system of the studied area.

Key words: Grazing - Biomass - Carrying capacity Woodland - Savanna - Cattle - Livestock management Benin.

\section{Resumen}

Agonyissa D., Sinsin B. Productividad y capacidad de carga de los pastizales naturales en Benin

El presente estudio se llevó a cabo en los pastizales sudanoguinenses del bosque clasificado de Wari Maro, en el centro de Benin. En base a los resultados obtenidos a partir de colectas fitosociológicas, se determinaron cuatro tipos de pastizales. Se trata de savanas herbáceas con Schizachyrium sanguineum y Sorghastrum bipennatum de las depresiones, de savanas de Andropogon schirensis y A. chinensis, de savanas de Pennisetum unisetum y de Chasmopodium caudatum y finalmente de tierras baldías con Pennisetum polystachion y Andropogon gayanus. La biomasa producida al final de la estación lluviosa varió de 3,45 $\pm 0,50$ a 5,19 $\pm 0,86$ t M S/ha. La biomasa más elevada se obtuvo en los pastizales con Schizachyrium sanguineum y Sorghastrum bipennatum de las depresiones. La capacidad de carga periódica después de los fuegos de monte se estimó en 0,16 unidades de ganado tropical por hectárea (UBT/ha), o sea una carga en la estación seca de 17200 UBT, para una superficie de 107500 ha. En la estación húmeda, el paso de los hatos y la explotación de los pastizales estuvieron ligados a la evolución de la fitomasa y a la disponibilidad de agua en el sistema de crianza extensivo utilizado en la zona estudiada.

Palabras clave: Pastoreo - Biomasa - Capacidad de carga Formación boscosa - Savana - Ganado bovino - Manejo del ganado - Benin. 